\title{
Endovascular surgery in Marfan syndrome: CON
}

\author{
Nicholas T. Kouchoukos
}

Division of Cardiovascular and Thoracic Surgery, Missouri Baptist Medical Center, BJC Healthcare, St. Louis, Missouri, USA

Correspondence to: Nicholas T. Kouchoukos, MD. 3023 N. Ballas Rd Suite 150D, St. Louis, Missouri 63131, USA. Email: ntkouch@aol.com.

\begin{abstract}
The frequency of endovascular stent grafting procedures to treat various conditions of the thoracic aorta has increased dramatically over the past three decades. Stent grafting has been applied on a limited basis in patients with Marfan syndrome and other connective tissue disorders, despite recommendations from current guidelines and expert consensus statements against its use in this setting. A review of publications reporting outcomes after stent grafting of the descending thoracic aorta in Marfan patients with acute or chronic aortic dissection indicates that these procedures can be accomplished with rates of early mortality, stroke and spinal cord ischemic injury that are comparable to those observed in patients who do not have Marfan syndrome. However, the rates of primary treatment failure (principally endoleak), secondary treatment failure, need for open repair and late death among the Marfan patients are substantially higher than those observed in patients without this condition. In addition, the rates of retrograde aortic dissection and development of stent-graft induced new entry (SINE), are also greater among patients with Marfan syndrome. All of these findings argue strongly against the routine use of endovascular grafts in Marfan patients with type B or residual type A dissection. Few data are available to assess the role of endografting in Marfan patients with aneurysmal disease, but the progressive aortic dilatation noted in these patients argues strongly against its use in this setting as well. At present, the available data indicate that there is no justification for elective stent grafting in Marfan patients with aortic dissection or aneurysm. The only reasonable indications for primary aortic stent grafting are in the setting of acute aortic dissection or rupture, where the intervention is considered lifesaving and rarely, considering the relatively young age of these patients, where the risk of open operation is considered to be prohibitive.
\end{abstract}

Keywords: Marfan syndrome; endovascular repair; aortic dissection; descending thoracic aorta; thoracic aortic aneurysm

Submitted Jun 06, 2017. Accepted for publication Oct 09, 2017.

doi: 10.21037/acs.2017.10.05

View this article at: http://dx.doi.org/10.21037/acs.2017.10.05

The frequency of endovascular stent grafting procedures on the thoracic aorta has increased dramatically over the past three decades. In many centers, stent grafting has become the treatment of choice for thoracic aortic aneurysm, dissection, coarctation and traumatic aortic disruption. It has also been used on a limited basis in patients with Marfan syndrome and other connective tissue disorders. At present, endovascular stent grafting for the latter group of patients is considered off-label and current guidelines and expert consensus statements do not recommend endovascular repair (1-3). This manuscript reviews the experience with endovascular stent grafting in Marfan syndrome focusing on patients with aortic dissection involving the descending thoracic aorta and provides evidence to support the recommendation that, with few exceptions, endovascular stent grafting for this condition is not advisable.

In 2013, Pacini and colleagues published a systematic review of studies reporting the early and mid-term results of endovascular stent grafting in patients with Marfan syndrome and type B aortic dissection (4). They identified 54 patients from 12 publications who had data suitable for analysis. The mean age of the patients was 40.9 years and approximately $75 \%$ were men (data for each of the variables examined in this review were not available for all patients). The majority had undergone a previous cardiovascular surgical procedure. Eleven of the patients (20.4\%) were 
treated for acute dissection and the remaining 43 for chronic dissection. Urgent or emergent procedures were performed in $30.6 \%$ ( 11 of 36 patients with available data). The proximal landing zone for the endovascular grafts was in the native aorta in $81 \%$ of the patients and in a previously implanted aortic graft in $19 \%$.

For the 40 patients reported in publications that focused only on patients with Marfan syndrome, the hospital mortality was $2.5 \%$ (one patient) and the stroke and spinal cord ischemic injury rates were $2.5 \%$ and $2.5 \%$ respectively (Table 1). No patient developed acute renal failure. Conversion to open operation was required in 2 $(5.0 \%)$ patients. Of interest, the single death and all of the above-mentioned complications occurred in the patients with chronic dissection. The peri-procedural endoleak rate for the 40 patients was $25.0 \%$ (10 patients) and 7 of the 10 endoleaks were type I. The endoleak rate was $30.3 \%$ when the patients who had the endograft positioned in a previously implanted graft were excluded from analysis. The early endoleak rate was substantially lower among the patients with acute dissection $(9.1 \%)$ than for those with chronic dissection (31\%), but the difference was not statistically significant.

In the follow-up interval that averaged approximately 2.5 years, endoleaks were observed in $17.9 \%$ of the 39 hospital survivors, open operation was required in $12.8 \%$ and a new endovascular procedure was performed in $17.9 \%$ (Table 1). A new dissection occurred in 1 of 17 patients (5.9\%) for whom follow-up information was available. New dissection, late endoleaks and need for additional endovascular procedures occurred only in patients presenting with acute dissection.

Pacini and colleagues also reviewed the available data regarding endovascular stent grafting of Marfan patients with non-dissecting aortic aneurysms (4). Only 12 patients were identified and the data were insufficient to draw meaningful conclusions.

Two additional publications have reported single institution experiences with endovascular grafting for patients with Marfan syndrome. Waterman and colleagues reported their experience with 16 patients (5). The pertinent clinical data are summarized in Table 1. Two patients had acute type B dissection, 11 had chronic type $\mathrm{B}$ dissection and three patients had other indications for intervention (two pseudoaneurysms, and one anastomotic disruption following open repair of an extensive thoracic aortic aneurysm). All 16 patients had undergone previous open aortic procedures (average of 1.9 per patient) and 14

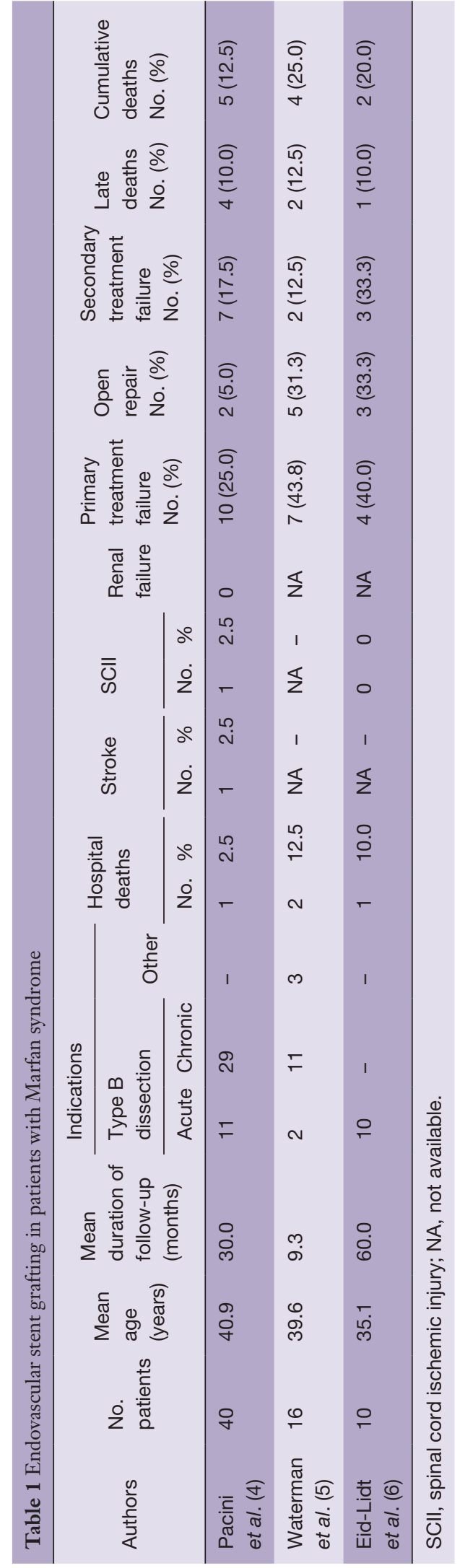


of the 16 were ASA class IV or IVE. Early mortality was $12.5 \%$ (two patients) and two additional patients died 1 and 3 months postoperatively, for a total mortality rate of $25.0 \%$. Seven patients $(43.8 \%)$ experienced primary treatment failure. Five of these patients underwent subsequent open repair, including one patient with retrograde dissection into the aortic arch. Three of the 4 deaths occurred in this primary failure group, for a mortality rate of $43 \%$. Two patients had secondary treatment failure. Both had persistent false lumen flow in the downstream aorta and subsequent aneurysmal degeneration. Both underwent subsequent endovascular procedures and one required additional open repair. Of note, 4 of the 6 patients who required subsequent open repair survived the procedure and were reported to be "alive and well", despite being initially considered at high risk for open repair.

Eid-Lidt and colleagues reported an experience with ten patients, all of whom presented with acute type $\mathrm{B}$ dissection with extension into the abdomen (DeBakey type IIIB) (6). Nine of the patients met "high risk" criteria for open repair, however, the criteria were not defined. One patient died 5 days after the endovascular procedure following open surgical repair for aortic rupture, for a hospital mortality rate of $10.0 \%$. One additional patient died approximately 9 months postoperatively, for a cumulative mortality rate of $20.0 \%$. The primary failure rate was $40.0 \%$ with two type I and two type II endoleaks. Three patients (33.3\%) required subsequent reinterventions for endoleak.

These studies indicate that although endovascular grafting procedures can be performed in Marfan patients with acceptable early mortality and morbidity rates, they are associated with early treatment failure rates of from $25.0 \%$ to $43.8 \%$ (primarily type I endoleaks) (Table 1 ). Open surgical repair was required in from $5 \%$ to $33 \%$ of patients in the early post-intervention period and secondary failure rates ranged from $12.5 \%$ to $33 \%$. As noted by Pacini and colleagues in their review (4), the rate of procedural endoleaks (i.e., primary treatment failures) observed in the 40 Marfan patients (25\%), was substantially higher than that reported in a systematic review of 567 patients with chronic type B dissection treated with endografts (7). Of the 309 patients available for analysis of this variable, the endoleak rate was $8.1 \%$ (25 patients). In the RESTORE patient registry of 76 patients with acute and chronic type B dissections treated with endografts, the endoleak rate was $7 \%$ for the acute and $8 \%$ for the chronic dissections (8). Kang and colleagues reported an early endoleak rate of $11.9 \%$ among 76 patients with chronic, complicated distal aortic dissection (9). The prevalence of Marfan syndrome among the patients in these three series ranged from $1.3 \%$ to $5.5 \%(7-9)$.

Additional problems associated with the use of endovascular stent grafts for patients with type B aortic dissection include retrograde aortic dissection and stentgraft induced new entry (SINE). Retrograde aortic dissection is a recognized complication of endografting of the descending thoracic aorta and the reported incidence has ranged from $1.4 \%$ to $20 \%$ (10). In a series of 443 patients undergoing endovascular stent grafting for acute, sub-acute and chronic type $\mathrm{B}$ aortic dissection reported by Dong and colleagues, 11 patients $(2.5 \%)$ developed a retrograde type A dissection (10). The retrograde dissections were detected from 2 hours to 36 months postoperatively. The early mortality rate for this complication was $27.3 \%$ (three patients). Three of the 11 dissections occurred in patients with known Marfan syndrome. Only 6 of the 443 patients in this study had Marfan syndrome and thus the frequency of retrograde dissection in these patients was $50 \%$. In their survey of the literature, Dong and colleagues noted that 5 of 37 patients (13.5\%) with retrograde aortic dissection had Marfan syndrome (10).

SINE is a major complication of endovascular stent grafting for type B aortic dissection. In 2010, Dong and colleagues reported the occurrence of SINE in 22 (3.4\%) of 650 patients undergoing endovascular stent-grafting for type B dissection (11). The prevalence of SINE among Marfan patients was $33.3 \%$ (2 of 6 patients). Weng and colleagues reported 27 distal SINE among 99 patients with residual type $\mathrm{A}$ or complicated type $\mathrm{B}$ aortic dissection, for a prevalence of $27 \%$ (12). SINE developed in 5 of 8 patients with Marfan syndrome (62.5\%). Eight of the 27 patients required secondary stent-grafting procedures and the remainder were treated conservatively.

Additionally, among patients who have undergone explantation of previously inserted descending thoracic aortic stent grafts, the percentage of patients with Marfan syndrome in the explanted graft cohort is substantially higher than the prevalence of Marfan syndrome in the original group receiving the stent graft. Ehrlich and colleagues, representing the European multicenter Medtronic Talent registry, reported on the short-term conversion rate to open surgery after endovascular stent grafting of the descending thoracic aorta (13). During a median interval of 14 months, 16 of 421 patients required explantation of the graft (15 for endoleak and 1 for retrograde type A dissection). Five of the 16 patients 
(31.3\%) had Marfan syndrome. However, Marfan patients represented only $3.6 \%$ of the total group (15 of 421 patients). By multivariable Cox analysis, Marfan syndrome was the most significant independent predictor of late conversion (adjusted hazard ratio 9.97, $\mathrm{P}=0.008$ ). In a series of reoperations for complications of endovascular procedures, reported by Spiliotopoulos and colleagues, 16 of 45 patients $(35.6 \%)$ who had a previous thoracic endograft had a connective tissue disorder (14 Marfan syndrome, 2 Loeys-Dietz syndrome) (14). Distal aortic dissection was present in all 16 patients.

All of the above findings argue strongly against the routine use of endovascular grafts in Marfan patients with type B or residual type A aortic dissection. Although the early mortality and morbidity rates are comparable to those for patients without Marfan syndrome, the Marfan patients have a greater prevalence of early endoleak (primary failure), need for open repair, secondary treatment failure, retrograde type A aortic dissection and SINE, in addition to poorer long-term outcomes.

Few data are available to assess the role of endografting in Marfan patients with aneurysmal disease, but the progressive aortic dilatation noted in Marfan patients, with the potential for separation of the graft from the enlarging aorta, argue strongly against its use in this setting as well.

At present, the available data indicate that there is no justification for elective endovascular stent grafting for patients with Marfan syndrome and type B or residual type A dissection, or with aneurysm in the absence of dissection. Highly satisfactory outcomes with open repair of Marfan patients with distal aortic disease have been reported in the last decade from several experienced surgical centers (15-19). Among a total of 219 patients undergoing open repair of thoracic or thoracoabdominal aneurysms (204 of whom had aortic dissection), early mortality ranged from $0 \%$ to $7 \%$, stroke from $0 \%$ to $5 \%$, renal failure from $0 \%$ to $13 \%$ and paraplegia from $0 \%$ to $7 \%$. In the largest series of 127 patients, 5-year survival was $87 \%$ and freedom from late repair failure $86 \%$ at 8 years (19).

An exception to this recommendation is the situation in which an endograft can be anchored both proximally and distally to previously placed synthetic grafts. Several studies have reported successful outcomes in patients who developed intercostal patch aneurysms after previous open repair of thoracic or thoracoabdominal aortic aneurysms where the stent grafts were anchored proximally and distally into the synthetic graft $(20,21)$.

The only other reasonable indications for endovascular stent grafting are in the setting of acute dissection or rupture, where there are clear indications for emergent intervention as a life-saving effort and the anatomy is suitable for graft implantation, or rarely, given the young age and generally good health of most Marfan patients, where the risk of open repair is considered to be prohibitive.

It is conceivable that newer, improved endografts may substantially reduce the frequency of or possibly eliminate the complications that are encountered with currently available grafts. Until this occurs and until clinical studies clearly document their safety and effectiveness, endovascular stent grafts should not be routinely used in patients with Marfan syndrome.

\section{Acknowledgements}

None.

\section{Footnote}

Conflicts of Interest: The author has no conflicts of interest to declare.

\section{References}

1. Svensson LG, Kouchoukos NT, Miller DC, et al. Expert consensus document on the treatment of descending thoracic aortic disease using endovascular stent-grafts. Ann Thorac Surg 2008;85:S1-41.

2. Hiratzka LF, Bakris GL, Beckman JA, et al. 2010 ACCF/AHA/AATS/ACR/ASA/SCA/SCAI/SIR/STS/ SVM Guidelines for the diagnosis and management of patients with thoracic aortic disease. A Report of the American College of Cardiology Foundation/American Heart Association Task Force on Practice Guidelines, American Association for Thoracic Surgery, American College of Radiology,American Stroke Association, Society of Cardiovascular Anesthesiologists, Society for Cardiovascular Angiography and Interventions, Society of Interventional Radiology, Society of Thoracic Surgeons,and Society for Vascular Medicine. J Am Coll Cardiol 2010;55:e27-129.

3. Erbel R, Aboyans V, Boileau C, et al. 2014 ESC Guidelines on the diagnosis and treatment of aortic diseases: Document covering acute and chronic aortic diseases of the thoracic and abdominal aorta of the adult. The Task Force for the Diagnosis and Treatment of Aortic Diseases of the European Society of Cardiology (ESC). Eur Heart J 
2014;35:2873-926.

4. Pacini D, Parolari A, Berretta P, et al. Endovascular treatment for type B dissection in Marfan syndrome: is it worthwhile? Ann Thorac Surg 2013;95:737-49.

5. Waterman AL, Feezor RJ, Lee WA, et al. Endovascular treatment of acute and chronic aortic pathology in patients with Marfan syndrome. J Vasc Surg 2012;55:1234-40; discussion 1240-1.

6. Eid-Lidt G, Gaspar J, Meléndez-Ramírez G, et al. Endovascular treatment of type $B$ dissection in patients with Marfan syndrome: mid-term outcomes and aortic remodeling. Catheter Cardiovasc Interv 2013;82:E898-905.

7. Thrumurthy SG, Karthikesalingam A, Patterson BO, et al. A systematic review of mid-term outcomes of thoracic endovascular repair (TEVAR) of chronic type B aortic dissection. Eur J Vasc Endovasc Surg 2011;42:632-47.

8. Zipfel B, Czerny M, Funovics M, et al. Endovascular treatment of patients with types A and B thoracic aortic dissection using Relay thoracic stent-grafts: results from the RESTORE Patient Registry. J Endovasc Ther 2011;18:131-43.

9. Kang WC, Greenberg RK, Mastracci TM, et al. Endovascular repair of complicated chronic distal aortic dissections: intermediate outcomes and complications. J Thorac Cardiovasc Surg 2011;142:1074-83.

10. Dong ZH, Fu WG, Wang YQ, et al. Retrograde type A aortic dissection after endovascular stent graft placement for treatment of type B dissection. Circulation 2009;119:735-41.

11. Dong Z, Fu W, Wang Y, et al. Stent graft-induced new entry after endovascular repair for Stanford type B aortic dissection. J Vasc Surg 2010;52:1450-7.

12. Weng SH, Weng CF, Chen WY, et al. Reintervention for distal stent graft-induced new entry after endovascular repair with a stainless steel-based device in aortic

Cite this article as: Kouchoukos NT. Endovascular surgery in Marfan syndrome: CON. Ann Cardiothorac Surg 2017;6(6):677-681. doi: 10.21037/acs.2017.10.05 dissection. J Vasc Surg 2013;57:64-71.

13. Ehrlich MP, Nienaber CA, Rousseau H, et al. Shortterm conversion to open surgery after endovascular stentgrafting of the thoracic aorta: the Talent thoracic registry. J Thorac Cardiovasc Surg 2008;135:1322-6.

14. Spiliotopoulos K, Preventza O, Green SY, et al. Open descending thoracic or thoracoabdominal aortic approaches for complications of endovascular aortic procedures: 19year experience. J Thorac Cardiovasc Surg 2017. [Epub ahead of print].

15. Dardik A, Krosnick T, Perler BA, et al. Durability of thoracoabdominal aortic aneurysm repair in patients with connective tissue disorders. J Vasc Surg 2002;36:696-703.

16. Kalkat MS, Rahman I, Kotidis K, et al. Presentation and outcome of Marfan's syndrome patients with dissection and thoraco-abdominal aortic aneurysm. Eur J Cardiothorac Surg 2007;32:250-4.

17. Mommertz G, Sigala F, Langer S, et al. Thoracoabdominal aortic aneurysm repair in patients with marfan syndrome. Eur J Vasc Endovasc Surg 2008;35:181-6.

18. Omura A, Tanaka A, Miyahara S, et al. Early and late results of graft replacement for dissecting aneurysm of thoracoabdominal aorta in patients with Marfan syndrome. Ann Thorac Surg 2012;94:759-65.

19. Coselli JS, Green SY, Price MD, et al. Results of Open Surgical Repair in Patients With Marfan Syndrome and Distal Aortic Dissection. Ann Thorac Surg 2016;101:2193-201.

20. Kulik A, Allen BT, Kouchoukos NT. Incidence and management of intercostal patch aneurysms after repair of thoracoabdominal aortic aneurysms. J Thorac Cardiovasc Surg 2009;138:352-8.

21. Schwill S, LeMaire SA, Green SY, et al. Endovascular repair of thoracic aortic pseudoaneurysms and patch aneurysms. J Vasc Surg 2010;52:1034-7. 